\title{
Adhesion of polydimethylsiloxane during molecular cross-linking
}

\author{
L. M. Dorogin ${ }^{\dagger, 1}$, I. M. Sosnin ${ }^{1,2}$, E. G. Akimov², V.I. Agenkov² \\ †leonid.dorogin.1984@gmail.com
}

${ }^{1}$ ITMO University, 49 Kronverksky Av., St. Petersburg, 197101, Russia

${ }^{2}$ Togliatti State University, 14 Belorusskaya St., Togliatti, 445020, Russia

\begin{abstract}
The transformation of polydimethylsiloxane (PDMS) material from a non-cross-linked liquid to a fully cross-linked solid state is investigated from the point of view of contact mechanics according to the classical Johnson-Kendall-Roberts scheme (JKR). The PDMS material sample was produced by mixing two liquids, namely PDMS base and cross-linking agent in a predefined ratio, and a further continuous measurement of the adhesion was performed during a chemical cross-linking reaction. It is demonstrated how adhesion between a glass ball and a PDMS sample is dependent on the PDMS cross-link density. The sample was in the liquid state for the first 16 hours. In the liquid state, the adhesive interaction can be quantitatively described as the result of the formation of a capillary bridge with an insignificant influence of the viscosity of the liquid up to the gel point. Then the sample turned into a soft gel-like structure, which corresponds to the formation of a percolated molecular network. It is shown that during the transformation of a liquid into a gel, its viscosity increases by several orders of magnitude ( 3 orders of magnitude in about 8 hours). The adhesive force as a function of time also shows a sharp increase when approaching the gel point of PDMS, where PDMS manifests itself as a tacky material, similar to pressure sensitive adhesives. During this transition period, the contact mechanics is characterized by the formation of "strings" and irreversible deformation, which are not observed either in the initial liquid state or in the completely bound state. Furthermore, the gel PDMS material continues to solidify, that is associated with changing of dynamical parameters of contact interaction as well as decreasing of adhesive "wear" of the PDMS surface.
\end{abstract}

Keywords: adhesion, viscoelasticity, cross-linking, polymer.

УДК: 661.718 .5

\section{Адгезия полидиметилсилоксана в ходе молекулярного связывания}

\author{
Дорогин Л.М., ${ }^{\dagger, 1}$ Соснин И. М., ${ }^{1,2}$, Акимов Е. Г. ${ }^{2}$ Агенков В. И. ${ }^{2}$ \\ †leonid.dorogin.1984@gmail.com \\ ${ }^{1}$ Университет ИТМО, Кронверкский пр., 49, Санкт-Петербург, 197101, Россия \\ ${ }^{2}$ Тольяттинский государственный университет, ул. Белорусская, 14, Тольятти, 445020, Россия
}

Исследуется превращение материала ПДМС из несвязанной жидкости в полностью связанное твердое состояние с точки зрения контактной механики по классической схеме Джонсона-Кендалля-Робертса (JKR). Образец материала ПДМС был получен смешиванием двух жидкостей - ПДМС-основы и связывающего реактива в заданной пропорции, и дальнейшего непрерывного измерения адгезии, происходящего в ходе химической реакции связывания. Показано, как адгезия между полидиметилсилоксаном (ПДМС) и стеклом зависит от плотности связывания молекулярной сети ПДМС. Образец находился в жидком состоянии первые 16 часов. В жидком состоянии адгезионное взаимодействие может быть количественно описано как результат образования капиллярного мостика с незначительным влиянием вязкости жидкости вплоть до момента гелеобразования. Далее, образец превратился в мягкую гелеобразную структуру, что соответствует образованию перколированной молекулярной сети. Показано, что в период превращения жидкости в гель ее вязкость возрастает на несколько порядков величины ( 3 порядка величины за $\approx 8$ часов). Сила адгезионного взаимодействия как функция времени также демонстрирует резкий рост при приближении к точке гелеобразования ПДМС, где ПДМС проявляет себя как очень липкий материал, 
подобный чувствительным к давлению адгезивам. В течение этого переходного периода контактная механика характеризуется образованием струн и необратимой деформацией, которые не наблюдаются ни в изначальном жидком состоянии, ни в полностью связанном состоянии. В дальнейшем гелеобразный материал ПДМС затвердевает, что сопровождается изменением динамических параметров контактного взаимодействия, а также снижением адгезионного «износа» поверхности ПДМС.

Ключевые слова: адгезия, вязкоупругость, связывание, полимер.

\section{1. Введение}

Взаимодействие между твердым шаром и плоской упругой подложкой часто описывается с помощью модели Джонсона-Кендалла-Робертса (JKR) [1]. Когда подложка является жидкостью, такое взаимодействие описывается капиллярной моделью. Обе модели основаны на минимизации полной энергии системы. Когда подложка представляет собой упругое тело, полная энергия состоит из энергии упругой деформации и энергии межфазного взаимодействия между атомами на контактирующей границе. Когда подложка является жидкостью, упругая энергия отсутствует, и энергия системы включает в себя только поверхностную и межфазовую энергии, а также потенциальную энергию гравитации. Для очень мягкого тела, например твердого вещества, близкого к точке геля, ни JKR, ни капиллярная модель не являются точными. Эта проблема может быть связана с другими эффектами, такими как деформация мягкой твердой поверхности из-за поверхностного натяжения [2-7]. Мы также отметим, что механика адгезии всегда включает в себя конечные временные интервалы, где неадиабатические эффекты могут быть важны и привести к гистерезису адгезивного контакта. Эти неадиабатические эффекты зависят от свойств материала, таких как вязкость жидкости и модули вязкоупругости, а также от молекулярных механизмов вблизи контактной трещины.

В этой статье мы сообщаем об экспериментах по адгезии с подложкой, которая непрерывно трансформируется из состояния жидкости в твердое состояние. В качестве подложки мы используем полидиметилсилоксан (ПДМС), в котором молекулы медленно связываются (cross-linking) в ходе химической реакции. В какой-то момент времени система достигает точки геля, где кластер связанных молекул образует перколированную сеть. В точке геля вязкость приближается к бесконечности, а статический модуль Юнга обращается в нуль. Однако при зондировании на конечных частотах материал обладает вязкоупругими свойствами [8].

ПДМС часто используются для модельных исследований адгезии и трения. Его можно легко получить с различной плотностью поперечных связей, используя различные соотношения между связывающим агентом и базовой жидкостью ПДМС [9].

Ниже мы демонстрируем адгезионные свойства материала ПДМС как функцию времени в процессе молекулярного связывания. Для этой цели мы подготовили несколько образцов ПДМС путем смешивания базовой жидкости ПДМС со связывающим агентом в соотношении $10: 1$, а затем мы изучили взаимодействие между стеклянным шаром и ПДМС, когда шар неоднократно приводился в контакт с образцом ПДМС.

\section{2. Материалы и методы}

Были изготовлены 5 образцов ПДМС из коммерческого двухкомпонентного набора Sylgard 184 (производство Dow Corning, США), состоящего из базовой жидкости (полидиметилсилоксан с виниловыми окончаниями) и связывающего агента (метилгидроксилоксан-диметилсилоксан кополимер) с подходящим катализатором. Из этих двух компонентов мы приготовили смесь в массовом отношении 10:1 (базовая жидкость/связывающий агент). Компоненты были перемешаны вручную в течении 1 мин., и далее смесь была дегазирована для удаления пузырьков воздуха, вызванных перемешиванием, затем вылита в чаши Петри. Образцы медленно затвердевали при комнатной температуре, в то же время они были помещены в экспериментальную установки для измерения адгезии. Так как вблизи точки геля образец ПДМС необратимо деформируется в ходе контакта, в ходе исследования мы заменяли образцы эквивалентными из той же партии образцов. Отметим, что перед взаимодействием со сферическим адгезионным зондом (стеклянным шаром) поверхности ПДМС очень гладкие из-за действия силы тяжести. Толщина слоя ПДМС составляла около 1 см, что намного больше диаметра области контакта, когда ПДМС находится в твердом состоянии. Следовательно, образцы ПДМС с точки зрения контактной механики можно считать полубесконечными.

Мы изучили адгезионное взаимодействие между стеклянным (soda-lime silica glass) шаром и образцом ПДМС с помощью той же установки, что использовалась нами ранее [9]. В ходе экспериментов мы приводим шар диаметром $2 R=2.5$ см в контакт с подложкой эластомера, как показано на Рис. 1. При переходе на новый образец ПДМС мы также заменили стеклянный шар новым чистым стеклянным шаром, так как стеклянные шары могут загрязняться при контакте с подложкой ПДМС. Подложка расположена на аналитических весах высокой точности (производство Mettler Toledo, модель MS104TS/00) с воспроизводимостью измерений в 0.1 мг (или $\approx 1 \mathrm{MH}$ ), которые служат в качестве датчика силы.

Чтобы перемещать стеклянный шар, мы использовали электрический шаговой двигатель, наматывающий нейлоновую леску, которая прикреплена к стеклянному шару. Скорость вытягивания как функция времени может быть задана на компьютере. В экспериментах, представленных ниже, стеклянный шар многократно перемещается вверх и вниз. В каждом цикле контакта сила нагрузки (когда ПДМС находится в твердом состоянии) составляет около $0.1 \mathrm{H}$, а вертикальная скорость $v_{\mathrm{z}} \approx 6 \mathrm{M \kappa м} / \mathrm{c}$.

Для измерений вязкости ПДМС 10:1 была приготовлена аналогичная смесь путем ручного смешивания компонентов в течении 4 минут. Вязкость смеси 
ПДМС измерялась при помощи инструмента Brookfield viscometer DV2T, используя различные скорости вращения шпинделя LV-04 (64), входящего в комплект инструмента, путем измерения сопротивления течения жидкости. Скорость вращения шпинделя изменялась в ходе набора вязкости в соответствии со стандартной процедурой производителя инструмента, и составила диапазон от 0.1 до 100 об/мин. Измерение вязкости, как и измерение адгезии протекало при комнатной температуре.

\section{3. Результаты}

На Рис. 2 показана сила взаимодействия между стеклянным шаром и поверхностью ПДМС как функция времени $t$, где $t=0$ соответствует времени, когда была приготовлена и разлита в образцы смесь ПДМС.

Для первого и второго образцов (красная и синяя кривые, соответствующие периодам времени 1-15 часов и 15-16 часов), ПДМС находился в жидком состоянии, причем их вязкость увеличивается со временем из-за увеличения плотности поперечных молекулярных связей. В течение этого периода времени нет отталкивающего взаимодействия между шаром и ПДМС, но формируется капиллярный мост притяжения между ними, как только стеклянный шар прикасается к поверхности жидкого ПДМС (Рис. 3).

Для жидкого мостика между двумя твердыми поверхностями сила взаимодействия $F_{\text {pull-off }}$ следует из уравнения Лапласа для отрицательного давления в капиллярном мостике, которое равно $p=-\gamma / r_{0}$, где $r_{0}$ - радиус кривизны мениска и $\gamma$ - поверхностное натяжение ПДМС (Рис. 3). Это приводит к соотношению

$$
F_{\text {pull-off }}=\pi r^{2}(-p)=\pi r^{2} \gamma / r_{0}
$$

где $r$ - радиус капиллярного моста, связанный с $r_{0}$. Из геометрических соображений высота капиллярного моста $h \approx r^{2} / 2 R$. Жидкость ПДМС смачивает поверхность стекла так, что $h=2 r_{0}$, таким образом $r_{0} \approx r^{2} / 4 R$.

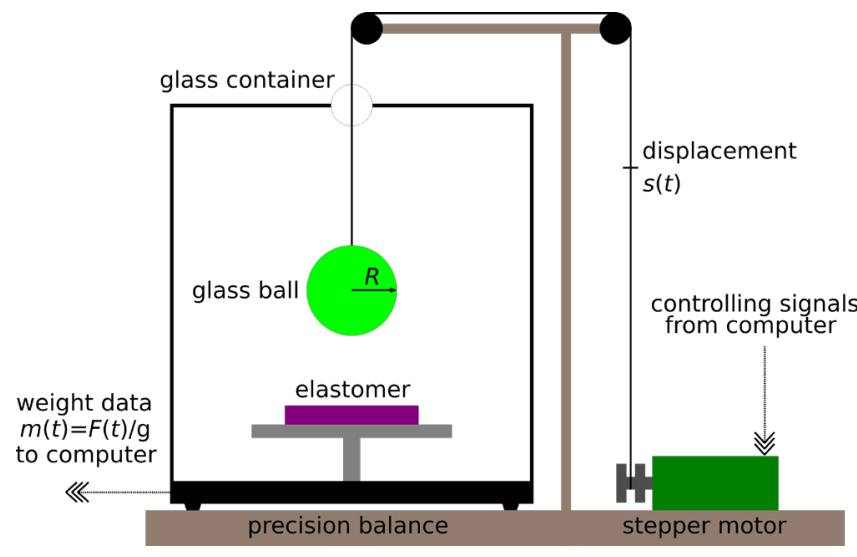

Рис. 1. Схема экспериментальной установки для измерения адгезии по методу JKR.

Fig. 1. Schematics of the experimental setup for measuring adhesion by JKR method.

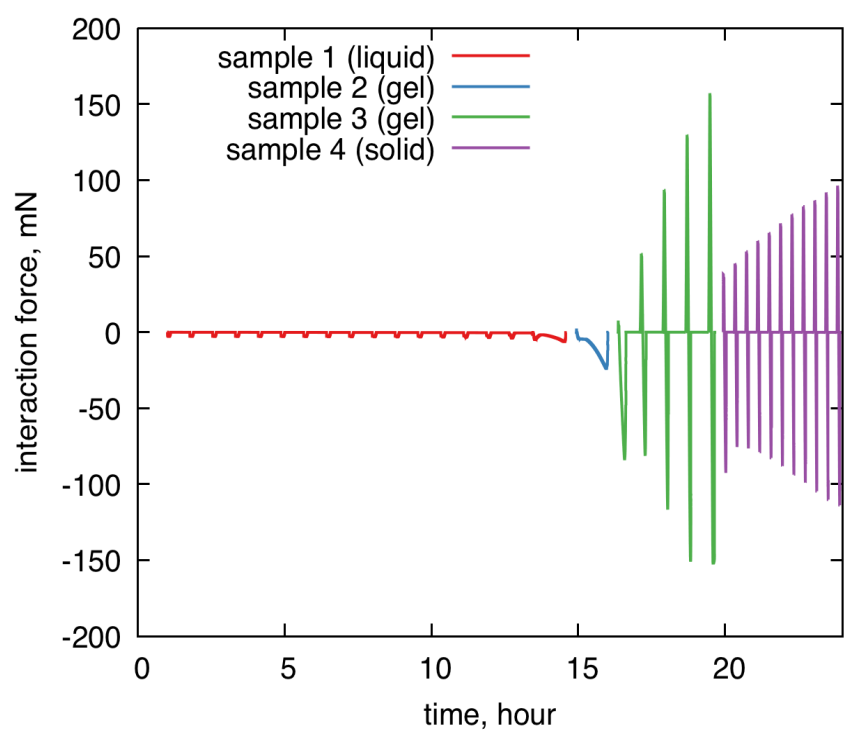

Pиc. 2. (Color online) Сила взаимодействия между образцом ПДМС и стеклянным шаром при повторяемых контактах как функция времени после подготовки образцов смеси ПДМС/связывающего агента 10:1 при $t=0$. Различные цвета показывают результаты, полученные для 4 разных образцов. Для первого образца (красная кривая, период времени 1-15 часов) ПДМС находится в жидком состоянии с вязкостью, увеличивающейся со временем. Для второго образца (синяя кривая) материал очень близок к точке гелеобразования. Для образца 3 (зеленая кривая) молекулярная сеть ПДМС достигла перколяции, и с момента первого контакта шара с ПДМС наблюдалась небольшая ненулевая сила отталкивания шара (положительная сила). Диаметр стеклянного шара $2 R=2.5$ см и скорость вытягивания $v_{z} \approx 5 \mathrm{Mкм} / \mathrm{c}$.

Fig. 2. (Color online) The interaction force between PDMS sample and the glass ball upon repeated contacts as a function of time after preparing a PDMS/cross-linker 10:1 mixture samples at $t=0$. The different colours show the results obtained for the 4 different samples. For the first sample (red curve; time period 1-15 hours), the PDMS is the liquid state with a viscosity increasing with time. For the second sample (blue curve), the material is very close to the gel point. For sample 3 (green curve) the molecular network has percolated and a small non-zero repulsive ball-PDMS interaction force (positive force) could be observed since the first ball-PDMS contact. The glass ball diameter $2 R=2.5 \mathrm{~cm}$ and the pull-off speed $v_{z} \approx 5 \mu \mathrm{m} / \mathrm{s}$.

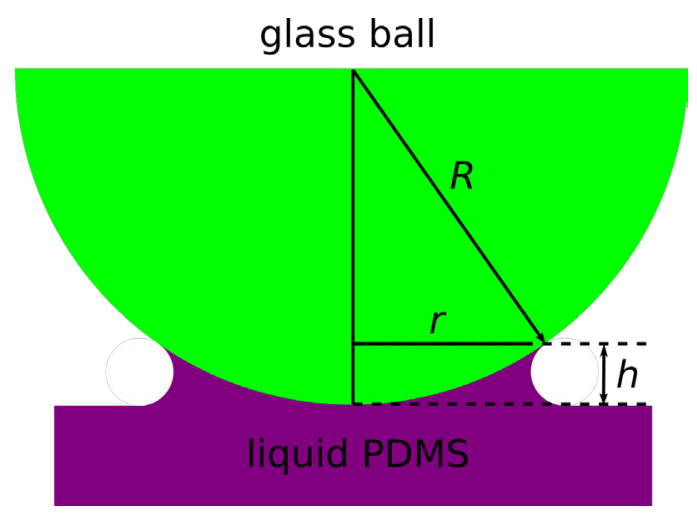

Рис. 3. Капиллярный мост, образованный в момент, когда стеклянный шар образует первоначальный контакт с жидким ПДМС.

Fig. 3. Capillary bridge formed at a point where the glass ball makes contact with the liquid PDMS. 
Используя это в формуле (1), получаем для силы взаимодействия [10]:

$$
F_{\text {pull-off }}=4 \pi R \gamma
$$

Для стеклянного шарика, контактирующего с жидкостью как в данном случае, картина более сложна, чем указано выше, и нужно учитывать влияние силы тяжести при изучении формы мениска. Эффект силы тяжести становится важным, когда число Бонда $B=\rho g h^{2} / \gamma>1$ [12], где $\rho$ и $\gamma$ - массовая плотность ПДМС и поверхностное натяжение, соответственно. В данном случае при $B \approx 10$, выражение (2) может, тем не менее, послужить грубой оценкой $\left(F_{\text {pull-off }} \approx 0.28 \mathrm{мH}\right.$ для образца 1 соответствует

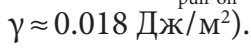

Заметим, что в течение последних нескольких циклов контакта для образца 1 сила вытягивания $F_{\text {pull-off }}$ оказалась выше, чем было предсказано по формуле (2), что мы объясняем влиянием возросшей вязкости жидкости. Для самого последнего контактного цикла на образце 1 процесс отрыва сильно подвержен влиянию вязкости жидкости, а фотографические снимки контакта вблизи к отрыву демонстрируют длинную нить ПДМС, прикрепленную к стеклянному шару (Рис. S2, дополнительный материал). Как известно, для жидкостей без вязкости (или в адиабатическом пределе, где вязкость не имеет значения) такая длинная жидкая нить неустойчива, и превратится в капли из-за поверхностного натяжения жидкости.

Для образца 2 (синяя кривая) материал очень близок к точке геля, в которой происходит перколяция молекулярной сети, образованной связанными олигомерами ПДМС. Точка геля характеризуется бесконечной вязкостью и обнулением модуля упругости, который при последующем затвердении становится конечной величиной. Мы также провели отдельные эксперименты для определения вязкости в процессе связывания как функции времени (Рис. 5), которые демонстрируют рост вязкости на 3 порядка величины (от $\sim 10^{3}$ до $\sim 10^{6}$ сСт) в течение 8 часов.

Для образца 3, где образовалась перколированная молекулярная сеть, небольшая сила отталкивания шара от подложки (положительная сила) наблюдается во время первого контакта (Рис. 2), и эта сила возрастает во время следующих контактных циклов.

На Рис. 4 показана работа, необходимая для отделения стеклянного шарика от подложки ПДМС в зависимости от времени после смешивания ПДМС. Работа по удалению шара была получена из площади под графиком на Рис. 2 под адгезионными провалами (где действует отрицательная сила взаимодействия), умноженная на скорость вытягивания $v_{z}$. Отметим резкий пик в работе отделения вблизи точки гелеобразования ПДМС.

Когда ПДМС находится в твердом состоянии, применяя модель JKR [1], работу адгезии $w$ можно получить из соотношения

$$
F_{\text {pull-off }}=\frac{3}{2} \pi R w .
$$

Это уравнение дает $w \approx 1.5$ Дж/м ${ }^{2}$ для образца 4 и $w \approx 0.4$ Дж/м² для образца 5 . Эти значения значитель-

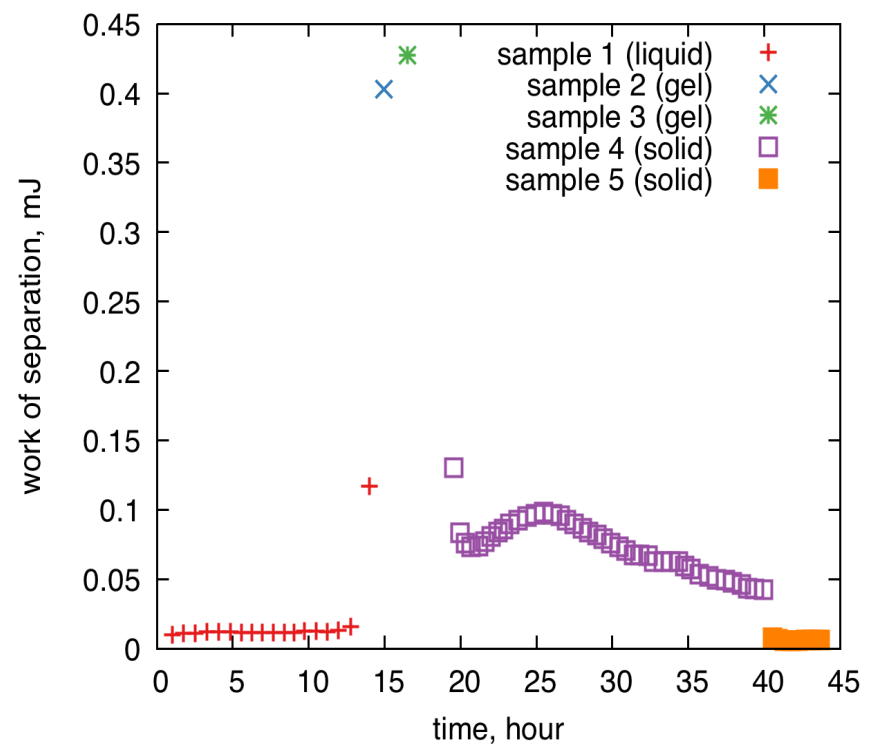

Рис. 4. Работа отделения стеклянного шара от поверхности ПДМС, показанная как функция времени, где время $t=0$ соответствует времени приготовления смеси ПДМС. Замечателен резкий пик в работе (образец 3) вблизи к точке гелеобразовния ПДМС.

Fig. 4. The work needed to separate the glass ball from the PDMS substrate is shown as a function of time, where time $t=0$ corresponds to the time the PDMS mixture was prepared. Note the sharp peak in the work (sample 3) close to the PDMS gel-point.

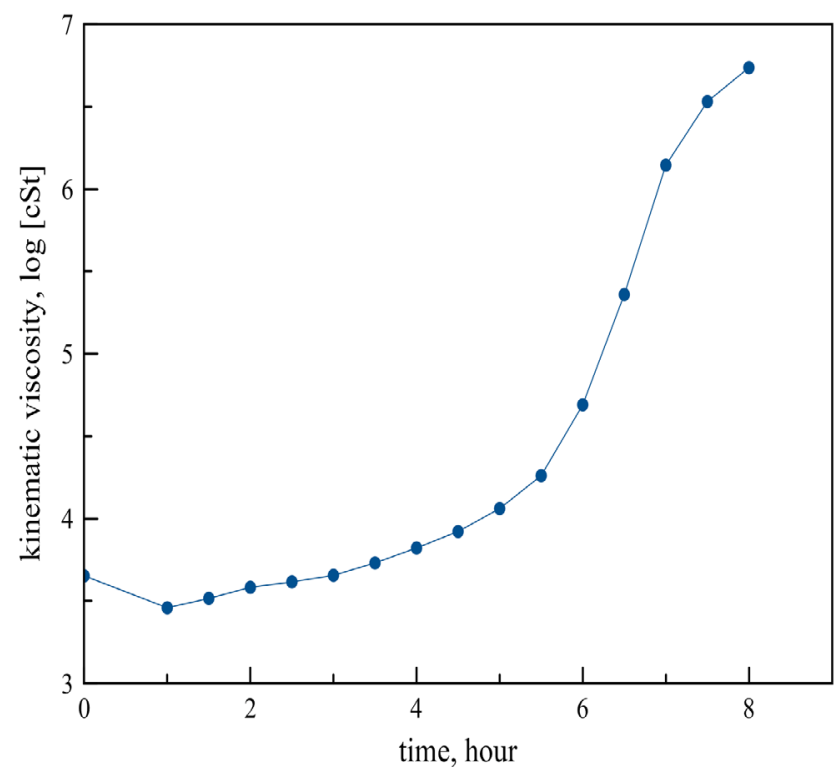

Рис. 5. Кинематическая вязкость, измеренная как функция времени, где время $t=0$ соответствует времени приготовления смеси ПДМС. В точке $t=0$ приведено измерение вязкости для чистой базовой жидкости ПДМС без связывающего агента. Дальнейшие измерения проведено для смеси ПДМС 10:1 при комнатной температуре.

Fig. 5. Kinematic viscosity measured as a function of time, where time $t=0$ corresponds to the time the PDMS mixture was prepared. At the moment $t=0$ the measurement was made for pure base PDMS liquid without cross-linking agent. Further measurements were conducted for PDMS 10:1 at room temperature. 
но выше, чем $w \approx 0.25$ Дж/ ${ }^{2}$ для ПДМС $10: 1$, связанные при повышенной температуре [9]. Мы объясняем это тем, что материал ПДМС образцов 4 и 5 не полностью связан, что имеет два последствия, а именно: (а) увеличивается внутреннее трение в материале, что приводит к существенному вязкоупругому вкладу в работу адгезии, и (б) образцы ПДМС необратимо деформируются в области контакта [13]. В результате поверхностных деформаций модель JKR не может быть строго применена в этих случаях. Работа адгезии даже через 30 дней $\left(w \approx 0.34\right.$ Дж/ $\left.\mathrm{M}^{2}\right)$ не достигла значение, которое ожидалось для полностью связанного ПДМС. Это показано на Рис. S1 (дополнительный материал), где последняя точка данных (синее перекрестие) для образца ПДМС после термообработки при $T=60^{\circ} \mathrm{C}$ в течение 24 часов, подготовленного за 30 дней до этого. Работа адгезии для этого образца $(w \approx 0.28$ Дж/м²) очень близка к тому, что было обнаружено в более ранних исследованиях при той же скорости отрыва для образцов, полученных с использованием аналогичной термообработки [9].

На Рис. S2 (дополнительный материал) показаны фотографические снимки во время последнего вытягивания для образца 1 (временной интервал $\approx 14-15$ часов). Отметим, что сила взаимодействия с шаром в момент (c) больше, чем в (b), несмотря на то, что нить может быть значительно тоньше, чем в точке (c). Ясно, что вязкоупругие напряжения в нити ПДМС должны быть значительно выше в случае (c), что может быть обусловлено, по крайней мере частично, более высокой вязкостью жидкости в момент (с).

Для образца 2 наблюдается похожее поведение, как и для последнего вытягивания на образце 1, но сила оказалась выше из-за более высокой плотности молекулярных связей и, следовательно, большей вязкости жидкости. Очевидно, что характер контактной механики для последнего вытягивания для образца 1 и для образца 2 сильно зависит от вязкости (или зарождающейся вязкоупругости) ПДМС.

Поверхность ПДМС деформируется после адгезионных измерений, проходящих вблизи точки гелеобразования (например, Рис. S2, d, дополнительный материал). Из-за необратимых деформаций поверхности ПДМС вблизи точки геля результаты за этот интервал времени в основном имеют качественный характер, но являются воспроизводимыми. Данные деформации по всей видимости не связаны с разрушением существующих перекрестных молекулярных связей, что подробнее описано в работе [13]. В жидком состоянии и в твердом состоянии вдали от точки геля не происходит необратимых деформаций поверхности материала.

На Рис. S3 (дополнительный материал) показана нить ПДМС образца 2, близком к моменту времени, когда притягивающее взаимодействие шара и ПДМС является максимальным (Рис. 2, отрицательный пик). Отметим образование множества тонких «струн» вблизи стеклянного шара, явления, которое часто наблюдается в чувствительных к давлению адгезивов [11]. Когда струны окончательно обрываются, на стеклянном шаре появляется узор, похожий на снежинку, и тонкая круглая пленка, как показано на Рис. S4 (дополнительный материал).

\section{4. Заключение}

В работе рассматривалась адгезия между стеклянным шаром и образцом ПДМС в зависимости от плотности поперечной молекулярной связи ПДМС, когда материал превращается из жидкого состояния в твердое состояние при комнатной температуре. Образец ПДМС получался путем смешивания базовой жидкости ПДМС и связывающего агента двухкомпонентного набора Sylgard 184 в соотношении 10:1. Образец ПДМС находился в жидком состоянии в течение первых $\approx 16$ часов, и в этом случае взаимодействие шара и ПДМС было чисто адгезионным, т.е. не наблюдалось никакого отталкивающего взаимодействия. В жидком состоянии это взаимодействие может быть количественно описано как результат образования капиллярного мостика с незначительным влиянием вязкости жидкости вплоть до момента гелеобразования. Показано, что в период превращения жидкости в гель ее вязкость возрастает на несколько порядков величины ( 3 порядка величины за $\approx 8$ часов).

Сила взаимодействия как функция времени демонстрирует резкий рост при приближении к точке гелеобразования ПДМС, где перекрестно связанные кластеры ПДМС перколируются, превращая жидкость в мягкое заполненное жидкостью поро-вязкоупругое вещество, и проявляет себя как очень липкий материал, подобный чувствительным к давлению адгезивам. В течение этого переходного периода контактная механика характеризуется образованием струн и необратимой деформацией, которые не наблюдаются ни в изначальном жидком состоянии, ни в полностью связанном состоянии. Для ПДМС в твердом состоянии контактная механика описывается моделью JKR, но до вплоть до момента 100 часов работа адгезии, полученная из модели JKR, все еще была больше, чем для полностью связанного ПДМС, описанного в [9]. Это согласуется с более ранними исследованиями материалов с меньшей концентрацией связывающего агента ПДМС $30: 1$ и 50:1, которые имеют большую долю несвязанных молекул ПДМС.

Благодарности/Acknowledgements. Данная работа выполнена в рамках проекта №18-19-00645 "Адгезия мягких материалов на основе полимеров: от жидкого состояния к твердому» при поддержке Российского Научного Фонда./This work was supported by Russian Science Foundation project grant 18-19-00645 "Adhesion of polymer-based soft materials: from liquid to solid".

Дополнительный материал/Supplementary Material. Электронная версия статьи содержит дополнительный материал (рисунки), доступный безвозмездно на сайте журнала (lettersonmaterials.com)./The online version of this paper contains supplementary material (figures and table) available free of charge at the journal's Web site (lettersonmaterials.com). 


\section{Литература/References}

1. K.L. Johnson, K. Kendall, and A.D. Roberts. Proc. R. Soc. London, Ser. A. 324, 301 (1971). DOI: 10.1098/rspa.1971.0141

2. R.W. Style, C. Hyland, R. Boltyanskiy, J. S. Wettlaufer, and E. R. Dufresne. Nat. Commun. 4, 2728 (2013). DOI: $10.1038 /$ ncomms3728

3. K.E. Jensen, R. Sarfati, R. W. Style, R. Boltyanskiy, A. Chakrabarti, M.K. Chaudhury, and E.R. Dufresne. Proc. Natl. Acad.Sci. U. S. A. 112, 14490 (2015). DOI: $10.1073 /$ pnas. 1514378112

4. R.W. Style, A. Jagota, C.Y. Hui and E. R. Dufresne. Annu. Rev. Condens. Matter Phys. 8, 99 (2017). DOI: 10.1146/annurev-conmatphys-031016-025326

5. O.D. Gordan, B. N.J. Persson, C.M. Cesa, D. Mayer, B. Hoffmann, S. Dieluweit, and R. Merkel. Langmuir. 24, 6636 (2008). DOI: 10.1021/la800728x
6. B. N. J. Persson, A. Kovalev, M. Wasem, E. Gnecco, and S. N. Gorb. EPL. 92, 46001 (2010).

7. A. Jagota, D. Paretkar and A. Ghatak. Phys. Rev. E: Stat., Nonlinear, Soft Matter Phys. 85, 51602 (2012). DOI: 10.1103/PhysRevE.85.051602

8. F. Chambon and H.H. Winter. J. Rheol. 31, 683 (1987).

9. A. Tiwari, L. Dorogin, A.I. Bennett, K. D. Schulze, W. G. Sawyer, M. Tahir, G. Heinrich and B. N. J. Persson. Soft Matter. 13, 3602 (2017). DOI: 10.1039/C7SM00177K

10. J. N. Israelachvili, Intermolecular and Surface Forces, 3rd edn, London, Academic (2011) 674 p.

11. C. Creton and M. Ciccotti. Rep. Prog. Phys. 79, 46601 (2016). DOI: 10.1088/0034-4885/79/4/046601.

12. P.-G. de Gennes, F. Brochard-Wyart, and D. Quere. Capillarity and Wetting Phenomena: Drops, Bubbles, Pearls, Waves, New York, Springer (2004) 292 p.

13. L. Dorogin and B. N. J. Persson. Soft Matter. 14, 1142 (2018). DOI: 10.1039/C7SM02216F 